\title{
Correlated terahertz acoustic and electromagnetic emission in dynamically screened InGaN/GaN quantum wells
}

van Capel, P. J. S.; Turchinovich, Dmitry; Porte, Henrik; Lahmann, S.; Rossow, U.; Hangleiter, A.; Dijkhuis, J. I.

Published in:

Physical Review B Condensed Matter

Link to article, DOI:

10.1103/PhysRevB.84.085317

Publication date:

2011

Document Version

Publisher's PDF, also known as Version of record

Link back to DTU Orbit

Citation $(A P A)$ :

van Capel, P. J. S., Turchinovich, D., Porte, H., Lahmann, S., Rossow, U., Hangleiter, A., \& Dijkhuis, J. I. (2011). Correlated terahertz acoustic and electromagnetic emission in dynamically screened InGaN/GaN quantum wells. Physical Review B Condensed Matter, 84(8), 085317. https://doi.org/10.1103/PhysRevB.84.085317

\section{General rights}

Copyright and moral rights for the publications made accessible in the public portal are retained by the authors and/or other copyright owners and it is a condition of accessing publications that users recognise and abide by the legal requirements associated with these rights.

- Users may download and print one copy of any publication from the public portal for the purpose of private study or research.

- You may not further distribute the material or use it for any profit-making activity or commercial gain

- You may freely distribute the URL identifying the publication in the public portal 


\title{
Correlated terahertz acoustic and electromagnetic emission in dynamically screened InGaN/GaN quantum wells
}

\author{
P. J. S. van Capel, ${ }^{1, *}$ D. Turchinovich, ${ }^{2}$ H. P. Porte, ${ }^{1,2}$ S. Lahmann, ${ }^{3}$ U. Rossow, ${ }^{3}$ A. Hangleiter, ${ }^{3}$ and J. I. Dijkhuis ${ }^{1}$ \\ ${ }^{1}$ Section Nanophotonics, Debye Institute for Nanomaterials Science, Department of Physics and Astronomy, University of Utrecht, \\ P.O. Box 80000, 3508 TA Utrecht, The Netherlands \\ ${ }^{2}$ DTU Fotonik - Department of Photonics Engineering, Technical University of Denmark, Ørsteds Plads 343, \\ DK 2800 Kongens Lyngby, Denmark \\ ${ }^{3}$ Institut für Angewandte Physik, Technische Universität Braunschweig, Mendelssohnstrasse 22, D-38106 Braunschweig, Germany
}

(Received 10 January 2011; revised manuscript received 8 June 2011; published 26 August 2011)

\begin{abstract}
We investigate acoustic and electromagnetic emission from optically excited strained piezoelectric $\mathrm{In}_{0.2} \mathrm{Ga}_{0.8} \mathrm{~N} / \mathrm{GaN}$ multiple quantum wells (MQWs), using optical pump-probe spectroscopy, time-resolved Brillouin scattering, and $\mathrm{THz}$ emission spectroscopy. A direct comparison of detected acoustic signals and $\mathrm{THz}$ electromagnetic radiation signals demonstrates that transient strain generation in InGaN/GaN MQWs is correlated with electromagnetic $\mathrm{THz}$ generation, and both types of emission find their origin in ultrafast dynamical screening of the built-in piezoelectric field in the MQWs. The measured spectral intensity of the detected Brillouin signal corresponds to a maximum strain amplitude of generated acoustic pulses of $2 \%$. This value coincides with the static lattice-mismatch-induced strain in $\mathrm{In}_{0.2} \mathrm{Ga}_{0.8} \mathrm{~N} / \mathrm{GaN}$, demonstrating the total release of static strain in MQWs via impulsive $\mathrm{THz}$ acoustic emission. This confirms the ultrafast dynamical screening mechanism in MQWs as a highly efficient method for impulsive strain generation.
\end{abstract}

DOI: 10.1103/PhysRevB.84.085317

PACS number(s): 72.20.Ht, 78.67.De, 62.30.+d, 63.22.Np

\section{INTRODUCTION}

Recent progress in the growth of $\operatorname{In}_{1-x} \mathrm{Ga}_{x} \mathrm{~N} / \mathrm{GaN}$ material systems allows the fabrication of a wide variety of single and multiple quantum well and superlattice structures with tailored band gaps, resulting in excellent lasers and light emitting diode devices in the blue and green spectral regions. ${ }^{1}$ Inherent to structures such as wurtzite piezoelectric InGaN/GaN quantum wells is the built-in strain due to lattice mismatch between the GaN barrier and the InGaN well material, causing an internal piezoelectric field reaching $\mathrm{MV} / \mathrm{cm}$ strengths. Ultrafast optical excitation of such structures leads to terahertz electromagnetic emission. ${ }^{2,3}$ Other experiments have shown that acoustic emission occurs as well. ${ }^{4-6}$ Recently, it was demonstrated that acoustic strain can be converted directly into $\mathrm{THz}$ radiation at the interface of a piezoelectric material. ${ }^{7}$

This study presents combined time-resolved THz electromagnetic emission spectroscopy and time-resolved (acoustic) Brillouin spectroscopy on InGaN/GaN multiple quantum well (MQW) samples with different QW thicknesses. ${ }^{2,3}$ The aim of these experiments is to make a quantitative connection between ultrafast screening of a built-in piezoelectric field, $\mathrm{THz}$ electromagnetic emission, and strain release.

\section{SAMPLES}

The MQW structures used in this work [Fig. 1(a)] are grown on a (0001)-oriented sapphire substrate by low-pressure metalorganic vapor-phase epitaxy. The crystallographic $c$-axis of $\mathrm{GaN}$ is oriented in the growth direction. First, a $2-\mu \mathrm{m}$ thick GaN buffer layer is grown on a sapphire substrate, followed by the MQW layer, and a $180-n m$ GaN cap layer. The MQW layer consists of ten identical $\mathrm{In}_{0.2} \mathrm{Ga}_{0.8} \mathrm{~N}$ quantum wells alternated by 7.2-nm thick GaN barriers. The three different samples used in this work have QW widths $L_{z}$ of 1.8, 2.7, and $3.6 \mathrm{~nm}$.
The $2-\mu \mathrm{m}$ thick GaN buffer layer between the quantum wells and the sapphire layer absorbs the strain associated with the lattice mismatch between $\mathrm{GaN}$ and sapphire. Therefore, the lattice mismatch between $\operatorname{In}_{0.2} \mathrm{Ga}_{0.8} \mathrm{~N} \mathrm{QW}$ and $\mathrm{GaN}$ barrier materials is the only source of strain inside the QWs. Given the lattice constants for wurtzite $\mathrm{GaN}^{8}$ and $\mathrm{InN},{ }^{9}$ and assuming a linear interpolation for the material compound mixture in the $\mathrm{QW}$, the relative difference in lattice constants between the QW and the barrier layer is around $1.9 \%$ for our samples in the direction of growth (c-direction).

Thus, the lattice mismatch between $\mathrm{InGaN}$ and $\mathrm{GaN}$ results in a built-in permanent strain in the QWs along the growth direction. This strain, combined with the large piezoelectric coefficient of InGaN, leads to the presence of a built-in electric field in the $\mathrm{QW}$, which for the case of $\operatorname{In}_{0.2} \mathrm{Ga}_{0.8} \mathrm{~N} / \mathrm{GaN}$ compounds reaches a strength of $3.1 \mathrm{MV} / \mathrm{cm}$ and is directed against the growth direction. ${ }^{10,11}$ Such a strong electric field in the QW results in the quantum-confined Stark effect (QCSE): ${ }^{12}$ a band structure tilt, a decrease of the optical transition energy, and a reduction of the optical transition strength.

\section{THZ ELECTROMAGNETIC AND ACOUSTIC EMISSION MECHANISMS}

$\mathrm{THz}$ electromagnetic emission from $\mathrm{InGaN} / \mathrm{GaN} \mathrm{QW}$ originates from ultrafast dynamics of electronic polarization $P$ in the biased QW, caused by the photoexcitation of polarized electron-hole pairs. ${ }^{13}$ The far-field emitted electric field $E_{\mathrm{THz}}(t)$ as a function of time $t$ is proportional to $\partial^{2} P / \partial t^{2}{ }^{13}$ Resonant optical excitation of an electron-hole pair in a QW subject to the QCSE results in a spatial separation of electrons and holes and a net transient electric dipole. The polarity of the dipole is opposite to that of the built-in piezoelectric field, leading to a (partial) screening of this field. In the case, when sufficient density of such optically excited dipoles is 
(a)

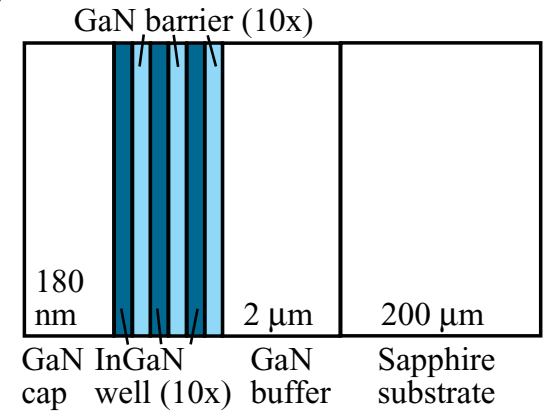

(b)

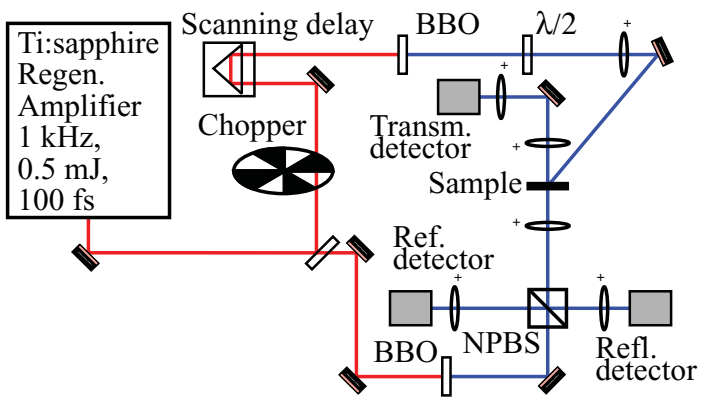

FIG. 1. (Color online) (a)Schematic picture of the multiple quantum well (MQW) samples, consisting of ten periods of GaN/InGaN material. (b) Experimental reflection/transmission setup.

excited by an ultrashort laser pulse [i.e. much faster than the typical recombination time (Ref. 10)], a significant or even complete screening of the initial built-in field can be achieved within the duration of the laser pulse (Refs. 2,3, and 14). This process is called the dynamical screening in biased quantum wells. The comprehensive general model of such a process was presented in Ref. 3, where polarization dynamics in the QWs and resulting $\mathrm{THz}$ emission is described for a wide range of excitation strengths: ranging from very weak excitation not leading to any sufficient built-in field screening, to extremely strong excitation leading to the complete removal of the built-in electric field (Ref. 2). The same model (Ref. 3 and references therein) allows for the treatment of the interaction of the ultrashort laser pulse with a biased QW as an effective $\chi^{(2)}$ nonlinear-optical process, with $\chi^{(2)}$ being the dynamical, time-dependent parameter of the net electric field in the QW at each moment in time during the excitation.

In terms of energy conservation, the dynamical screening of a biased QW can be viewed as a coherent discharge of a nanoscale capacitor, where the electrostatic energy initially stored in a biased QW is released via free-space $\mathrm{THz}$ electromagnetic emission. Dynamical screening is accompanied by a blueshift in the QW photoluminescence as the Stark-shift resulting from initially present strong QCSE vanishes, and by an increase in the spatial overlap of electron and hole wave functions. This process is described in detail in our earlier works. $^{2,3}$

As soon as the electric field within the piezoelectric QW becomes screened, the mechanical stress in the $\mathrm{QW}$ is no longer balanced by the electrostatic force. This can be viewed as an "inverse" piezoelectric effect, i.e., the change in electric field leading to a change in lattice deformation. The timescale of the resulting strain release is determined by the QW thickness of several nanometers divided by the sound velocity. The sound velocity in $\mathrm{GaN}$ is around $8 \mathrm{~km} / \mathrm{s},{ }^{15}$ and we estimate the longitudinal sound velocity in $\mathrm{In}_{0.2} \mathrm{Ga}_{0.8} \mathrm{~N}$ at $7.4 \mathrm{~km} / \mathrm{s}$ by linear interpolation of the sound velocities in $\mathrm{GaN}$ and InN. ${ }^{16}$ At these velocities, the typical travel times through the 1.8 and $3.6 \mathrm{~nm}$ wells are 240 and $480 \mathrm{fs}$, respectively. The timescale of strain release therefore lies in the subpicosecond domain. Acoustic (sub-) THz emission was observed in Refs. 4 and 17 for QWs, and in Ref. 18 for quantum dots. In case of (long) picosecond pulse excitation, typical emitted strain amplitudes are calculated to exceed values of $0.1 \%$, which makes this mechanism far more efficient in strain generation than the deformation potential electron-phonon interaction also occurring in nanostructured systems. ${ }^{19}$

In this work, we will demonstrate a direct and quantitative correlation between electromagnetic and acoustic $\mathrm{THz}$ emission from dynamically screened piezoelectric InGaN/GaN MQWs.

\section{EXPERIMENT}

We used $\mathrm{THz}$ emission spectroscopy for the investigation of $\mathrm{THz}$ electromagnetic emission and pump-probe reflection/transmission spectroscopy at $400 \mathrm{~nm}$ wavelength to examine the $\mathrm{THz}$ acoustic emission from the MQWs. The heart of our experimental setup is a femtosecond Ti:sapphire regenerative amplifier operating at $1 \mathrm{kHz}$ repetition rate, and producing pulses of about $120 \mathrm{fs}$ duration at $800 \mathrm{~nm}$ central wavelength. The frequency-doubled $400 \mathrm{~nm}$ excitation pulses had a similar duration as the original Ti:sapphire output pulses, and were generated by second-harmonic generation in a $\beta$-barium borate crystal. This wavelength is above band gap in the MQWs (both in the Stark-shifted and fully screened cases), but below the band gap energy of GaN (barrier) corresponding to a wavelength of $360 \mathrm{~nm}$.

\section{A. Terahertz emission spectroscopy}

Terahertz electromagnetic emission is associated with the transient polarization in the QWs, directed along the bias field, i.e., normal to the sample surface. The samples were rotated at $\sim 45^{\circ}$ angle with respect to the excitation beam, in order to provide a nonzero component of the transient dipole in the direction perpendicular to the excitation beam. ${ }^{2}$ The temporal dependency of the electric field in the emitted $\mathrm{THz}$ pulses was phase-sensitively detected using standard free-space electrooptic sampling in a $1-\mathrm{mm}\langle 110\rangle$-oriented ZnTe crystal. The sampling beam with the central wavelength of $800 \mathrm{~nm}$ was derived from a fundamental output of the Ti:sapphire amplifier. A detailed description of a $\mathrm{THz}$ emission experiment can be found in Refs. 2 and 13.

Typical THz signals for the 3.6-nm MQW are shown in Fig. 2(a) in the pump fluence range $0.02-1.3 \mathrm{~mJ} / \mathrm{cm}^{2}$. The spectrum of the detected $\mathrm{THz}$ pulses, covering the range 0-3 THz, is limited by the corresponding acceptance bandwidth of the $1-\mathrm{mm} \mathrm{ZnTe} \mathrm{THz} \mathrm{detector} \mathrm{crystal} \mathrm{at} 800 \mathrm{~nm}$ 

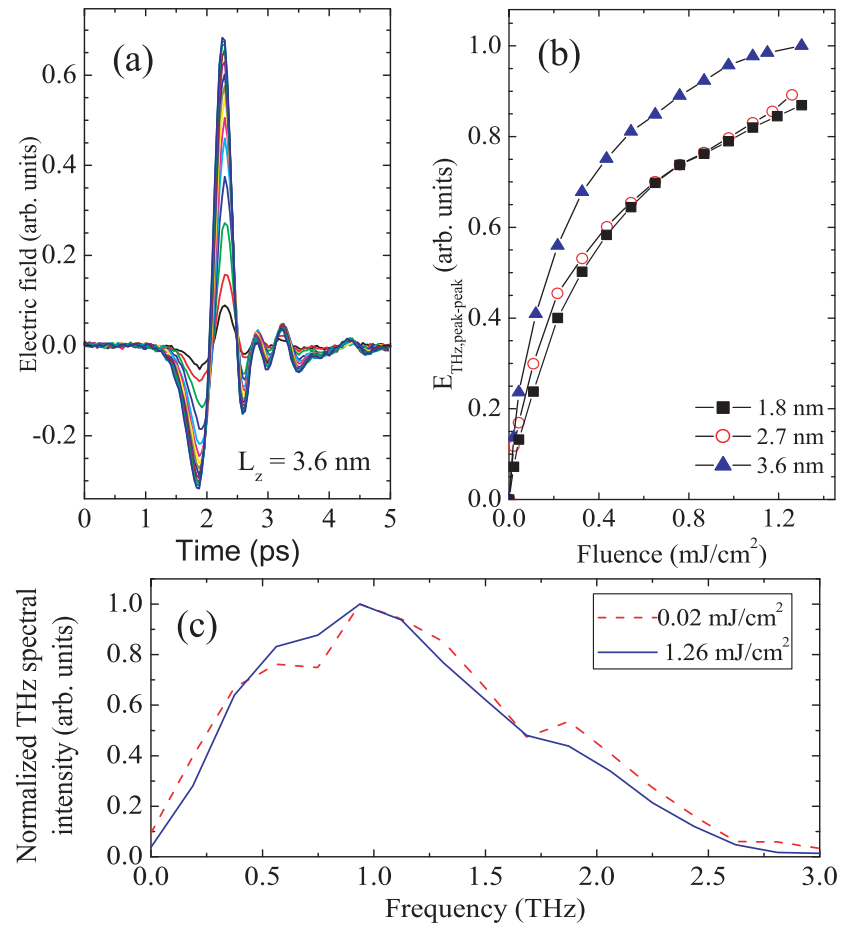

FIG. 2. (Color online) (a) Measured THz signals as a function of pump fluence in the range $0.02-1.26 \mathrm{~mJ} / \mathrm{cm}^{2}$, for the 3.6-nm MQW sample. (b) THz peak-peak amplitudes versus fluence for all samples. (c) Normalized $\mathrm{THz}$ spectral amplitudes at 0.02 and $1.26 \mathrm{~mJ} / \mathrm{cm}^{2}$, for the $L_{z}=3.6 \mathrm{~nm}$ MQW sample.

sampling wavelength, as illustrated by the normalized amplitude spectra of a weak and very strong $\mathrm{THz}$ pulse emitted by the sample with $L_{z}=3.6 \mathrm{~nm}$. The $\mathrm{THz}$ output monotonously grows with increasing pump fluence until saturation becomes apparent at around $0.5 \mathrm{~mJ} / \mathrm{cm}^{2}$.

The determined $\mathrm{THz}$ peak field strength (in arbitrary units) as a function of fluence for all samples is shown in Fig. 2(b). The MQW sample with $L_{z}=3.6 \mathrm{~nm}$ produces a roughly $30 \%$ larger $\mathrm{THz}$ electric field emission than the other two samples in the fluence range used. Saturation sets in at the fluence of $\sim 0.5 \mathrm{~mJ} / \mathrm{cm}^{2}$ for all three samples with $L_{z}=1.8,2.7$, and $3.6 \mathrm{~nm}$. The saturation of $\mathrm{THz}$ output is explained by the depletion of the electrostatic energy in the initially biased QW (acting as a nano-scale capacitor) by excitation of a substantial density of polarized electron-hole pairs. At full bias field screening, all electrostatic energy is released, and the symmetry in the QW is restored.

\section{B. Pump-probe measurements at $400 \mathrm{~nm}$ wavelength}

The pump-probe setup is shown in Fig. 1(b). The pump beam is chopped to allow for differential measurements. Polarizations of pump and probe beams were set orthogonal using a half-wave plate, and polarization filters were inserted to suppress pump light at the detectors. The pump beam is focused to a beam waist of $170 \mu \mathrm{m}$, with a fluence on the sample surface in the range $0.1-20 \mathrm{~mJ} / \mathrm{cm}^{2}$. The probe beam was focused down to $15 \mu \mathrm{m}$, and the probe fluence was kept below $0.1 \mathrm{~mJ} / \mathrm{cm}^{2}$. The $800-\mathrm{nm}$ pulse length was measured at $180 \mathrm{fs}$. Typical pump and probe pulse durations after frequency
TABLE I. Reflection, transmission, and absorption for the used QW samples. Values were determined for $400 \mathrm{~nm}$, and at low fluence.

\begin{tabular}{lccc}
\hline \hline QW thickness $L_{z}$ & $R$ & $T$ & $\alpha$ \\
\hline $1.8 \mathrm{~nm}$ & 0.29 & 0.55 & 0.16 \\
$2.7 \mathrm{~nm}$ & 0.11 & 0.53 & 0.36 \\
$3.6 \mathrm{~nm}$ & 0.15 & 0.44 & 0.41 \\
\hline \hline
\end{tabular}

doubling are estimated at $\tau_{p} \sim 180 / \sqrt{2}=130$ fs. Relative changes in reflection $\Delta R / R$ and transmission $\Delta T / T$ were measured simultaneously. The separately measured absolute reflection coefficient $R$ and transmission coefficient $T$ at a very low fluence of $0.2 \mathrm{~mJ} / \mathrm{cm}^{2}$ are collected for each sample in Table I. The absolute values allow for a calculation of the relative changes in absorption $\Delta \alpha / \alpha$, where the absorption $\alpha=1-R-T$.

We note that the reflection mainly consists of the sample surface reflection. Higher order reflection contributions (at the $\mathrm{GaN} /$ sapphire interface and at the sapphire surface) are small and further neglected in the analysis.

$\Delta T / T$ and $\Delta R / R$ for the MQW sample with $L_{z}=$ $3.6 \mathrm{~nm}$ are shown for a range of fluences in Figs. 3(a) and 3(b), respectively. The traces for the MQW samples with $L_{z}=$ $1.8 \mathrm{~nm}$ and $L_{z}=2.7 \mathrm{~nm}$ look similar and are not shown here. The detailed plot of $\Delta R / R$ in Fig. 3(b) reveals clear Brillouin oscillations, associated with the acoustic vibrations in the MQW structure. These will be analyzed later on in this paper.

The calculated absorption $\Delta \alpha / \alpha$ for the 3.6-nm MQW is shown in Fig. 3(c). The maximum transient absorption $\Delta \alpha_{\max }$ at time $t=0$ as a function of pump fluence for the three different samples is presented in Fig. 3(d). The change in optical properties of the sample directly after pumping is related to the optical excitation of hot carriers in the MQWs. The absorbed pump light $I_{\text {pump }}$ increases the population of excited electrons and holes by $\Delta n$, which in turn proportionally reduces the probe absorption $\Delta \alpha$. We can thus assume that $\Delta \alpha_{\max }$ is a measure for pump absorption. From Figs. 3(d) and 2(b), we observe that the absorption efficiency $\Delta \alpha_{\max }$ as well as the generated $\mathrm{THz}$ electric field amplitudes for the $L_{z}=$ $3.6 \mathrm{~nm} \mathrm{MQW}$ are higher by $\sim 30 \%$ compared to both the $L_{z}$ $=2.7 \mathrm{~nm}$ MQW and the $L_{z}=1.8 \mathrm{~nm}$ MQW. We therefore conclude that acoustic emission in the $\mathrm{THz}$ range would be approximately equal for all samples at equal absorption.

The maximum normalized absorption $\Delta \alpha_{\max } / \alpha$ is shown in the inset of Fig. 3(c) for the pump fluence range 0-3 $\mathrm{mJ} / \mathrm{cm}^{2}$. The pump absorption is linear for all samples in the pump fluence range below $2 \mathrm{~mJ} / \mathrm{cm}^{2}$ [Fig. 3(d), inset]. The onset of saturation of optical effects in Fig. 3 at a fluence of $2 \mathrm{~mJ} / \mathrm{cm}^{2}$ is due to bleaching of the optical transition. Saturation of electromagnetic $\mathrm{THz}$ emission, however, sets in already at fluences $<1 \mathrm{~mJ} / \mathrm{cm}^{2}$, see Fig. 2(b). This demonstrates as expected that carrier generation continues when the piezoelectric field is already completely screened. ${ }^{3}$

After pump excitation at $t=0$, the signal decays nonexponentially, with a typical $(1 / e)$ decay time decreasing to $30 \mathrm{ps}$ for the highest pump fluences. The dynamics is governed by carrier decay under the conditions of restoring QCSE, and the decay times are consistent with dynamic conductivity measurements on the same samples. ${ }^{20}$ 

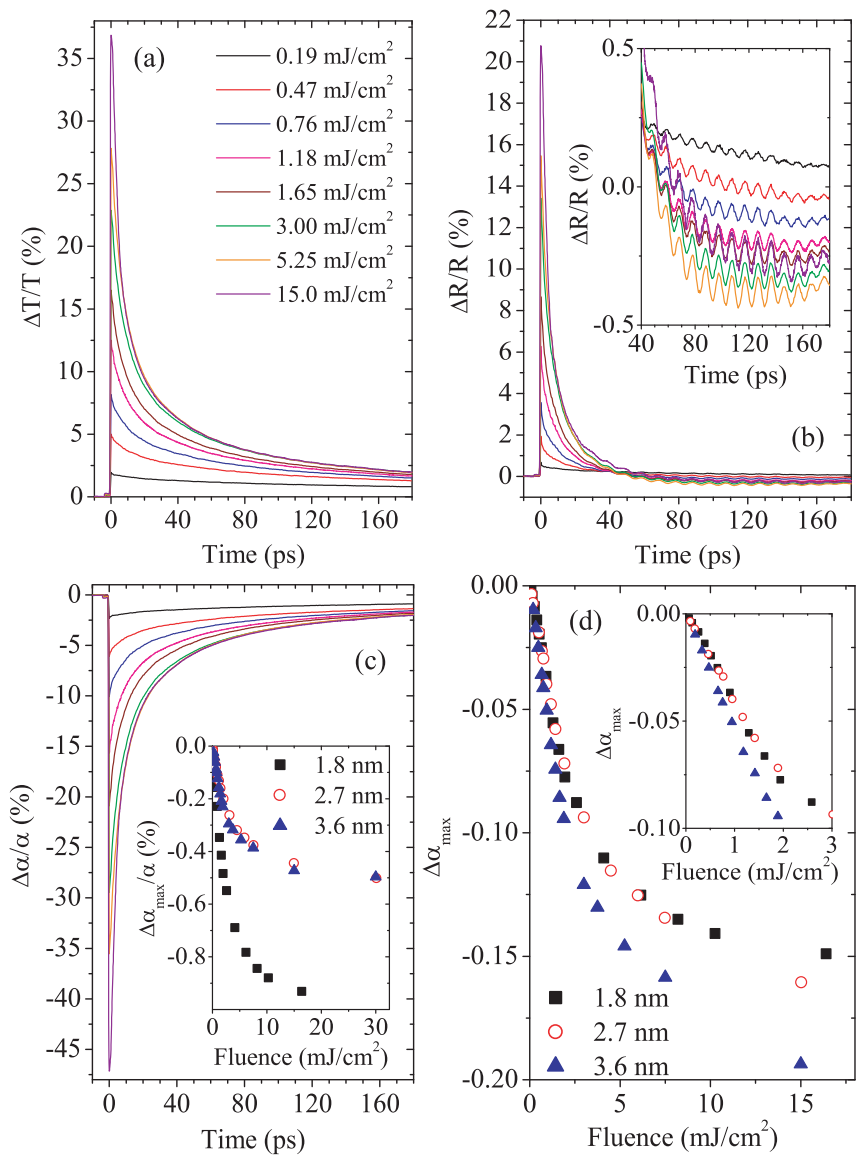

FIG. 3. (Color online) (a) Time-dependent transmission changes of the 3.6-nm thick MQW for a range of pump fluences. (b) Timedependent reflection changes of the 3.6-nm thick multiple quantum well for the same pump fluences as (a). (Inset) Detail showing Brillouin oscillations. (c) Time-dependent relative absorption changes for the 3.6-nm thick multiple quantum well for the pump fluences in (a). Inset shows maximum relative change in absorption $\Delta \alpha_{\max } / \alpha$ as a function of pump fluence, for the three MQWs. (d) Maximum amplitude of absolute change in absorption at $t=0\left(=\alpha_{\max }\right)$ as a function of pump fluence. Inset shows $\alpha_{\max }$ for fluences $<3 \mathrm{~mJ} / \mathrm{cm}^{2}$ and demonstrates that absorption is linear for the lowest fluences.

\section{Signatures of zone-folded acoustic phonons}

Since optical absorption occurs periodically in the individual QWs in the MQW on a nanometer length scale, THz electromagnetic emission should be accompanied by emission of a periodic train of ultrashort acoustic THz pulses. The bandwidth of excited phonon frequencies is limited to $\sim 2 \mathrm{THz}$ by the $<500 \mathrm{fs}$ time required for sound to travel through the quantum well structure. Because the acoustic impedance contrast of the $\mathrm{GaN}$ barrier and $\mathrm{InGaN}$ well is low, the generated strain may escape from the MQW region efficiently. The periodic structure of the MQW does cause zone-folding of the phonon dispersion relation within the first Brillouin zone. ${ }^{21,22}$ This implies that terahertz phonon modes do indeed form in the MQW at low wave vector $k$. These modes can be populated and observed both in the optical reflection and the transmission. We will use these zone-folded modes to further characterize our samples.
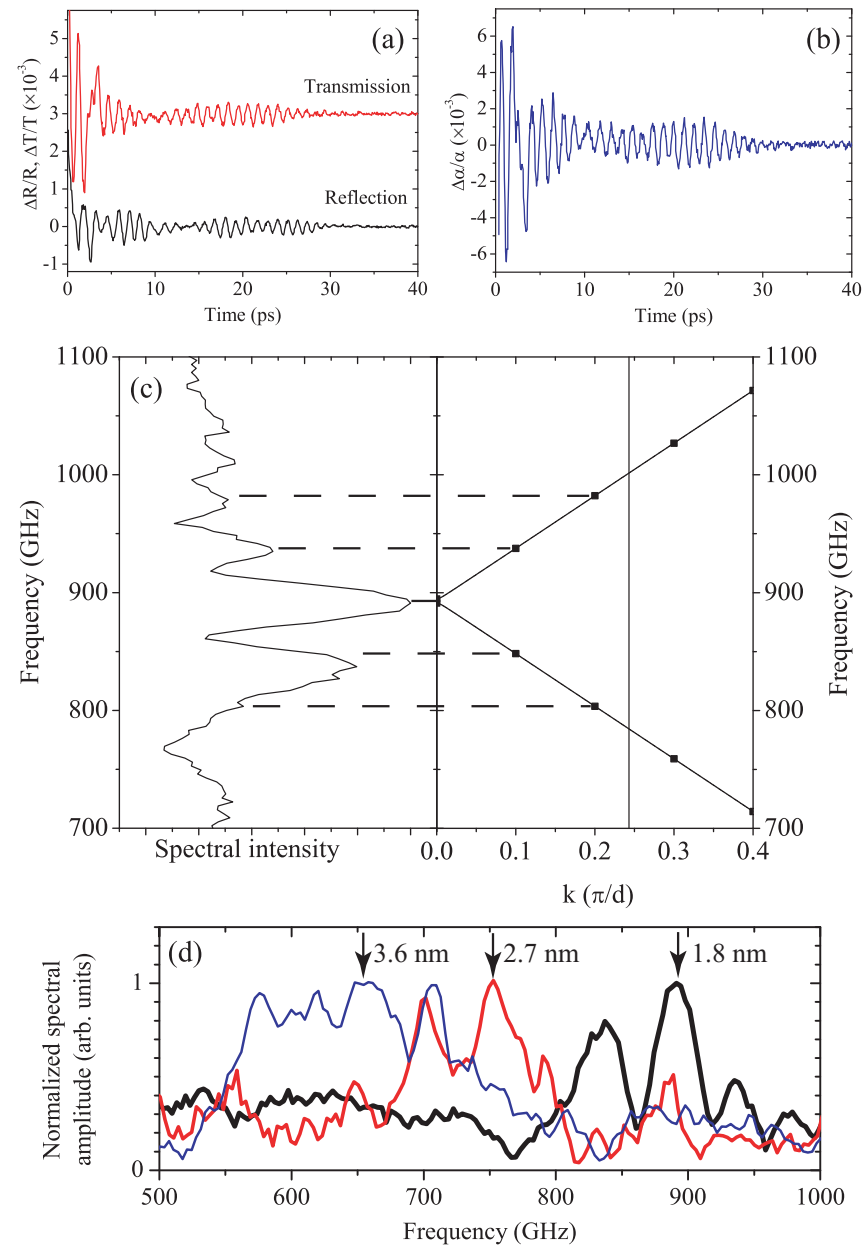

FIG. 4. (Color online) (a) Background-subtracted reflection and transmission for short times for the 1.8-nm MQW and a pump fluence of $25 \mathrm{~mJ} / \mathrm{cm}^{2}$. (b) Absorption modulation $\Delta \alpha / \alpha$ due to coherent superlattice vibrations, derived from (a). (c) (Left panel) Phonon spectrum of the 1.8-nm superlattice vibrations obtained by Fourier transformation of the signal in (b). (Right panel) Calculated dispersion curve. Here, squares indicate the calculated discrete superlattice modes. (d) Phonon spectra of absorption modulations for $1.8-\mathrm{nm}$ (thick line), 2.7-nm (medium thick line) and 3.6-nm (thin line) MQW samples. Arrows indicate $k=0$ mode positions.

In Fig. 4(a), we show the differential reflection and transmission for the 1.8-nm MQW and a pump fluence of $25.6 \mathrm{~mJ} / \mathrm{cm}^{2}$, after subtracting the slow decaying background from the original data by taking a moving average of the original trace over the oscillation period. ${ }^{23}$ At this high pump fluence, the piezoelectric field inside the MQW is already fully screened. ${ }^{2,3}$ Coherent vibrations of the phononic superlattice with a period of $1.5 \mathrm{ps}$ and a characteristic beating pattern are observed during the first $30 \mathrm{ps}$.

The results for the differential absorption corresponding to the signals in Fig. 4(a) are shown in Fig. 4(b). The absorption modulation originates from the acoustic modulation of the carrier density. The decay time of $14 \mathrm{ps}$ is determined by the acoustic escape time from the MQW (i.e., roughly the total MQW thickness divided by the average sound velocity), shorter than the carrier lifetime of $\sim 30$ ps (cf. Fig. 3). 
To analyze the measured fast oscillations in the pump-probe trace, we applied a Fourier transform to the measured pumpprobe trace after subtracting the slow background. The Fourier amplitudes of the time-domain signal in Fig. 4(b) are shown in Fig. 4(c), left panel. The observed peaks correspond to the allowed discrete vibrational modes at $k=(n / N) \times(\pi / d)$, with $N=10$ the number of QWs, $n$ ranging from 1 to $N$, and $d$ is the total thickness of the superlattice. The short duration of the signal caused by propagation of the acoustic energy into the QW surroundings results in broad spectral modes. The calculated zone-folded acoustic phonon mode positions for the 1.8-nm thick quantum well are indicated by squares in Fig. 4(c), right panel. The frequency of the highest $(n=2)$ mode reaches $1000 \mathrm{GHz}$.

Normalized spectra are shown in Fig. 4(d) for all three MQW samples. The expected shift of the $k=0$ modes to lower frequencies for thicker quantum wells is evident. The higher order modes can be observed for MQWs with $L_{z}=$ $2.7 \mathrm{~nm}$ and $L_{z}=3.6 \mathrm{~nm}$, but they are less pronounced for the MQW with $L_{z}=1.8 \mathrm{~nm}$, due to a lower signal-to-noise ratio in these measurements. The $k=0$ modes (indicated by arrows) for the 2.7- and 3.6-nm samples are 10\% lower in frequency than expected from calculations, most likely due to slight uncertainties in layer thicknesses and actual speed of sound.

We have thus clearly demonstrated acoustic superlattice mode emission from dynamically screened $\mathrm{InGaN} / \mathrm{GaN}$ MQWs in the $\mathrm{THz}$ range. Unfortunately, the low signal-tonoise ratio did not allow for a direct quantitative comparison of the acoustic signal strength versus fluence with the results for electromagnetic emission in the previous section. Instead, we use Brillouin measurements and monitor inelastic light scattering off the acoustic pulse as it propagates through the sample.

\section{Signatures of Brillouin oscillations}

The impulsive stress in the MQW stack, arising from partial or complete screening of the built-in piezoelectric field, is transferred to the surrounding GaN layers of the sample in the form of coherent acoustic wave packets. ${ }^{24}$ A single welldefined Fourier component of such a wave packet can be conveniently monitored by time-resolved Brillouin scattering. ${ }^{25,26}$

In such an experiment, the acoustic wave locally changes the refractive index of the material, creating a condition for a weak probe reflection interfering with static probe reflections off the surface of the sample, the quantum well structure, and the GaN/sapphire interface. Since the acoustic wave moves, a periodic reflection variation emerges with the Brillouin frequency $f_{0}=2 n v_{s} / \lambda$ for the case of perpendicular probing. Here, $n$ is the refractive index of the material, $v_{s}$ is the sound velocity, and $\lambda$ is the probe wavelength. Brillouin frequencies $f_{0}=107 \mathrm{GHz}$ in $\mathrm{GaN}$ and $f_{0}=100 \mathrm{GHz}$ in sapphire, respectively, result for $\lambda=400 \mathrm{~nm}$. Note that in the present case of a buried strain generator, such as a QW, two separate acoustic wavefronts are formed, leading to multiple interferences. For interference between probe beam reflections from the moving acoustic wave and from the stationary interfaces, a spatiotemporal overlap of these reflections is required. The coherence time $t_{\text {coh }}$ (the time in which the overlap decreases with a factor of $1 / e$ ) is given by $t_{\mathrm{coh}}=c \tau /\left(2 \sqrt{\ln 2} n v_{s}\right)$, with $\tau$ the optical pulse temporal full
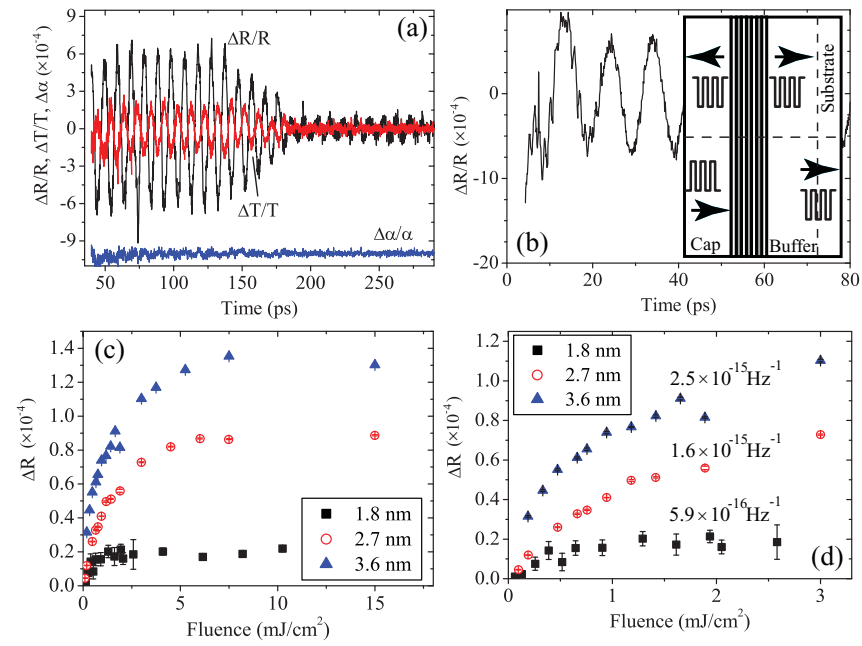

FIG. 5. (Color online) (a) Brillouin oscillations in reflection $\Delta R / R$ and transmission $\Delta T / T$ for the 3.6-nm thick multiple quantum well and an excitation fluence of $15 \mathrm{~mJ} / \mathrm{cm}^{2}$. Between 140 and $180 \mathrm{ps}$, acoustic waves travel from the GaN buffer layer into the sapphire substrate. Bottom line is absorption $\Delta \alpha / \alpha$ calculated from reflection and transmission data, displaced by $-10^{-3}$. (b) Brillouin oscillations for the $2.7-\mathrm{nm}$ thick multiple quantum well at an excitation fluence of $10.5 \mathrm{~mJ} / \mathrm{cm}^{2}$ shortly after excitation. Before 22 ps (left of dashed line), generated acoustic waves travel in opposite directions (inset, upper panel). One contribution travels through the cap layer and reflects at the free surface (inset, lower panel). After $22 \mathrm{ps,} \mathrm{both} \mathrm{contributions} \mathrm{travel} \mathrm{in} \mathrm{the} \mathrm{same} \mathrm{direction.}$ (c) Brillouin oscillation amplitudes as a function of fluence, in the range $0-15 \mathrm{~mJ} / \mathrm{cm}^{2}$. (d) Oscillation amplitudes in the range $0-3 \mathrm{~mJ} /$ $\mathrm{cm}^{2}$. Numbers indicate the spectral amplitudes at the saturation fluence of $1.5 \mathrm{~mJ} / \mathrm{cm}^{2}$ in $\mathrm{Hz}^{-1}$. These spectral amplitudes are derived from a comparison of the measured Brillouin oscillations to measurements on a propagating pulse with a given spectrum.

width at half-maximum. ${ }^{25}$ For a $400-\mathrm{nm}$ probe pulse of $130 \mathrm{fs}$, we find $t_{\mathrm{coh}}=1.1 \mathrm{~ns}$. Decrease of interference can therefore be ignored on the relevant timescales $<200$ ps of our experiment.

The Brillouin oscillation amplitude is determined by the spectral amplitude of the Brillouin frequency in the wave packet, and the elasto-optic coupling parameter of the material. Since the generated pulse spectrum is determined largely by the width of the MQW stack, we take the Brillouin frequency amplitude as a measure for the generated strain, thereby making a direct comparison with $\mathrm{THz}$ electromagnetic emission feasible.

The ratio of the two amplitudes reflects the $T / R$ ratio of 1:3 (Table I). Since no net absorption takes place in the inelastic scattering process, an increase in reflection should correspond to a decrease in transmission, and vice versa. Indeed, reflection and transmission in Fig. 5(a) are in opposite phase, and the absorption $\Delta \alpha / \alpha$ is shown to be near zero.

The measured time-dependent amplitude of the Brillouin oscillation can be explained from the sample geometry. In Fig. 5, three different regimes can be distinguished, related to acoustic wave propagation sketched in Fig. 5(b), inset. The reflection signal at earlier times, i.e., when the generated Brillouin contributions travel in opposite directions, is shown for the 2.7-nm quantum well in Fig. 5(b). About 20 ps after arrival of the pump pulse, the amplitude of the Brillouin oscil- 
lation changes. This corresponds to the travel time $(180 \mathrm{~nm} /$ $7.8 \mathrm{~nm} / \mathrm{ps}=23 \mathrm{ps}$ ) of one of the acoustic pulses to the surface through the 180 -nm thick GaN cap layer. Upon reflection of this component, the interference with the component emitted in the direction of the substrate changes. Actually, reflection occurs between 15 and 30 ps because of the total thickness of the MQW region of $\sim 100 \mathrm{~nm}$. Between 40 and $140 \mathrm{ps,}$ the component reflected at the cap layer and the component emitted in the direction of the substrate travel through GaN in the same direction (toward the sapphire substrate). Since these components travel at equal speed and thus at constant distance to each other, the amplitude of the Brillouin oscillation remains constant. Between 140 and 185 ps, the oscillation is decreasing. Here, the acoustic wave emitted inward reaches the sapphire substrate, while the other returning from the cap layer still travels in the $2-\mu \mathrm{m}$ thick $\mathrm{GaN}$ buffer layer. The amplitude decreases slowly because it takes time for the two contributions (separated by twice the thickness of the cap layer, i.e., $360 \mathrm{~nm}$ ) to fully enter the sapphire substrate. After $180 \mathrm{ps,}$ both acoustic contributions travel through sapphire, in which the elasto-optic coupling is much smaller compared to $\mathrm{GaN}$, as is the observed Brillouin signal amplitude. ${ }^{24}$

In order to quantify the measured results, the Brillouin oscillations in the reflection signals are fitted in the range 40$140 \mathrm{ps}$ with a sinusoidal function. The fitted average Brillouin frequency of $103.3 \pm 0.2 \mathrm{GHz}$ in $\mathrm{GaN}$ is only slightly lower than the calculated value $107 \mathrm{GHz}$, and points to a fractionally lower sound velocity or refractive index than estimated earlier.

In order to obtain the absolute reflectivity variation $\Delta R$ due to a single Brillouin contribution, corrections have to be made to the measured amplitudes for the different reflection coefficients $R$ of Table I, and interference between the two traveling contributions [Fig. 5(b), inset].

We have further corrected for sample-to-sample variations in cap layer thickness as derived from the fitted Brillouin oscillation offset. It is likely that such variations are partly responsible for the variations in absolute reflection and transmission (Table I). The oscillation amplitudes found in the reflection signal after correction, are plotted in Fig. 5(c) as a function of pump fluence for the three MQW samples. A detailed plot of the region $0-3 \mathrm{~mJ} / \mathrm{cm}^{2}$ is shown in Fig. 5(d).

Two fluence regimes can clearly be distinguished in this plot: a low-fluence acoustic generation regime for which saturation sets in around $0.5 \mathrm{~mJ} / \mathrm{cm}^{2}$ (i.e., the same fluence at which the saturation in $\mathrm{THz}$ electromagnetic emission sets in), and a high-fluence acoustic generation, showing complete saturation around $10 \mathrm{~mJ} / \mathrm{cm}^{2}$, except for the thinnest sample, which shows only a weak increase in Brillouin intensity above $1.5 \mathrm{~mJ} / \mathrm{cm}^{2}$. The high-fluence saturation behavior of the measured Brillouin amplitudes follows the optical absorption saturation $\Delta \alpha$ shown in Fig. 3(d). This high-fluence contribution is likely due to the weaker process of strain generation via deformation potential electron-phonon interaction. ${ }^{19}$ The efficiency of the latter process is ultimately limited by bleaching of the quantum well.

We have calibrated the spectral intensities $S\left(f_{0}\right)_{\text {sapph }}$ at the Brillouin frequency by comparing the measured Brillouin oscillation $\Delta R$ to the measured Brillouin oscillations for a known pulse shape generated in a chromium film and propagating through sapphire. ${ }^{27,28}$ The ratio between the oscillation amplitudes in GaN and sapphire [Fig. 5(a)], determined by the ratio in elasto-optic constants, ${ }^{24}$ can readily be used to connect the spectral intensity in GaN $S\left(f_{0}\right)_{\mathrm{GaN}}$ and $\Delta R$.

We were thus able to establish that a value for $\Delta R$ of $10^{-4}$ measured in GaN corresponds to a value of $S\left(f_{0}\right)_{\mathrm{GaN}}=$ $(3.0 \pm 0.8) \times 10^{-15} \mathrm{~Hz}^{-1}$. We will use this value to estimate the absolute strain amplitudes generated by the screening process for the three MQW samples. The absolute values for $S\left(f_{0}\right)_{\mathrm{GaN}}$ corresponding to $\Delta R$ at the saturation fluence of acoustic generation by screening of $1.5 \mathrm{~mJ} / \mathrm{cm}^{2}$ are indicated for the three MQW samples in Fig. 5(d).

\section{DISCUSSION}

We have established that acoustic (Fig. 5) and THz electromagnetic (Fig. 2) emission signals from dynamically screened piezoelectric InGaN/GaN MQWs are strongly correlated. Both kinds of emission start to saturate at pump fluences around $0.5 \mathrm{~mJ} / \mathrm{cm}^{2}$ at which full screening of the built-in piezoelectric bias field takes place in the MQWs, as determined from the vanishing Stark shifts in the photoluminescence. ${ }^{2}$ We plot the measured $\Delta R$ due to acoustic emission against the THz signal strength for pump fluences up to $1.5 \mathrm{~mJ} / \mathrm{cm}^{2}$ in Fig. 6. The perfectly linear relation between the $\mathrm{THz}$ electromagnetic and acoustic output confirms that electromagnetic emission and acoustic $\mathrm{THz}$ emission have a common origin in piezoelectric field screening.

We note here that at the weakest pump fluence, around $0.05 \mathrm{~mJ} / \mathrm{cm}^{2}, \mathrm{THz}$ electromagnetic emission is still observable, while Brillouin signal originating from the acoustic emission is not detected. What is the cause of the threshold behavior visible in Fig. 6 for all MQW samples?

The most likely reason for this offset is the strong nonlinearity of the dynamical screening effect on the pump fluence. $^{3}$ At the weakest excitation, the polarized electron and hole states are weakly populated, which still results in the polarization dynamics and hence $\mathrm{THz}$ electromagnetic emission, but no substantial bias field screening occurs. Such a situation for weak pump is described in detail in Ref. 13. The acoustic emission, however, is directly related to bias

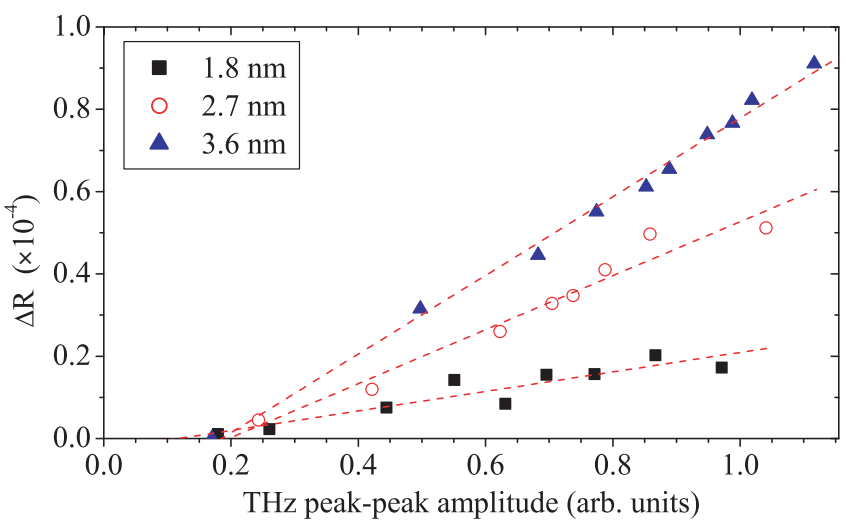

FIG. 6. (Color online) Brillouin amplitudes versus $\mathrm{THz}$ signal amplitudes, for fluences below $1.5 \mathrm{~mJ} / \mathrm{cm}^{2}$. Dashed lines are linear fits to the data. The linear relation demonstrates that acoustic emission and electromagnetic emission are strongly correlated. 
field screening since relaxation of lattice strain occurs only when the electrostatic force of the built-in field does not compensate the mechanical stress in the piezoelectric QW anymore. Therefore, the lattice dynamics becomes observable only at sufficiently strong pump fluences when the built-in field becomes sufficiently screened.

The absolute spectral amplitude for the acoustic $100 \mathrm{GHz}$ component $S\left(f_{0}\right)_{\mathrm{GaN}}$ is related both to the shape of the MQW and the initial strain amplitude $s_{0}$. We will now show that the spectral intensities at the Brillouin frequency observed under the condition of complete field screening, Fig. 5(d), allow us to estimate the initial amplitude of the emitted coherent strain pulses. To this end, we calculate strain propagation for a strain pulse that is generated upon full screening of the piezoelectric field inside the QW.

In the work of Sanders and Stanton, ${ }^{19}$ a detailed modeling of carrier dynamics in the quantum wells is performed to calculate the generated propagating strain shape $s(z, t)$ due to both the deformation potential and the piezoelectric screening. ${ }^{19}$ In our case, we can make some reasonable assumptions to avoid such a detailed approach. First, since our pump pulse duration $\tau_{p} \sim$ $130 \mathrm{fs}$ is much shorter than the acoustic travel times through the quantum well estimated at $<500 \mathrm{fs}$, we may assume that the strain is generated instantaneously. Since $\tau_{p}$ is a factor of 8 shorter than the value of 1 ps used by Sanders, we expect the generated phonon components to have higher frequencies. Second, we assume that the deformation potential contribution [Fig. 5(c)] is negligible below $1.5 \mathrm{~mJ} / \mathrm{cm}^{2}$. Third, calculations of electron and hole wave functions demonstrate that for this case, carrier densities are reduced at the edges of the quantum well, due to finite carrier localization. ${ }^{2,3}$ We therefore assume that full screening for each quantum well is achieved in a region $\sim 1 \mathrm{~nm}$ narrower than the quantum well thickness.

The propagation of strain $s(z, t)$ through the full sample structure is then calculated by numerically solving the wave equation

$$
\frac{\partial^{2} s}{\partial t^{2}}=\frac{\partial^{2}}{\partial z^{2}}\left(v_{s}^{2} s\right)
$$

with $v_{s}=v_{s}(z)$ the material-dependent sound velocity. The amplitude $s_{0}$ for the square-packet initial strain is left as free parameter to fit the measured spectral intensities.

The calculated acoustic wave propagating in the GaN buffer layer after $30 \mathrm{ps}$ and corresponding phonon spectra are shown in Fig. 7 for the three samples. The superlattice mode structure of the MQW (Fig. 4) determines the phonon spectrum. The spectral intensities around the Brillouin frequency $f_{0}$ closely match the values of Fig. 5(d). We obtain a good agreement between the measured and the calculated spectral intensities by using values for $s_{0}$ of $2.0 \%, 1.8 \%$, and $1.7 \%$ for the $1.8,2.7$, and $3.6 \mathrm{~nm}$ samples, respectively, with a relative accuracy of $25 \%$. This large relative error is mainly caused by the indirect method for obtaining the spectral amplitudes $S\left(f_{0}\right)_{\mathrm{GaN}}$, and to a minor extent by our assumptions about the generated strain shape. These values for $s_{0}$ are nevertheless in a very good agreement with the value of $1.9 \%$ determined from the lattice mismatch between the QW and the barrier.

We thus conclude that the full built-in strain is released fully and coherently upon complete screening of the built-in piezoelectric field.
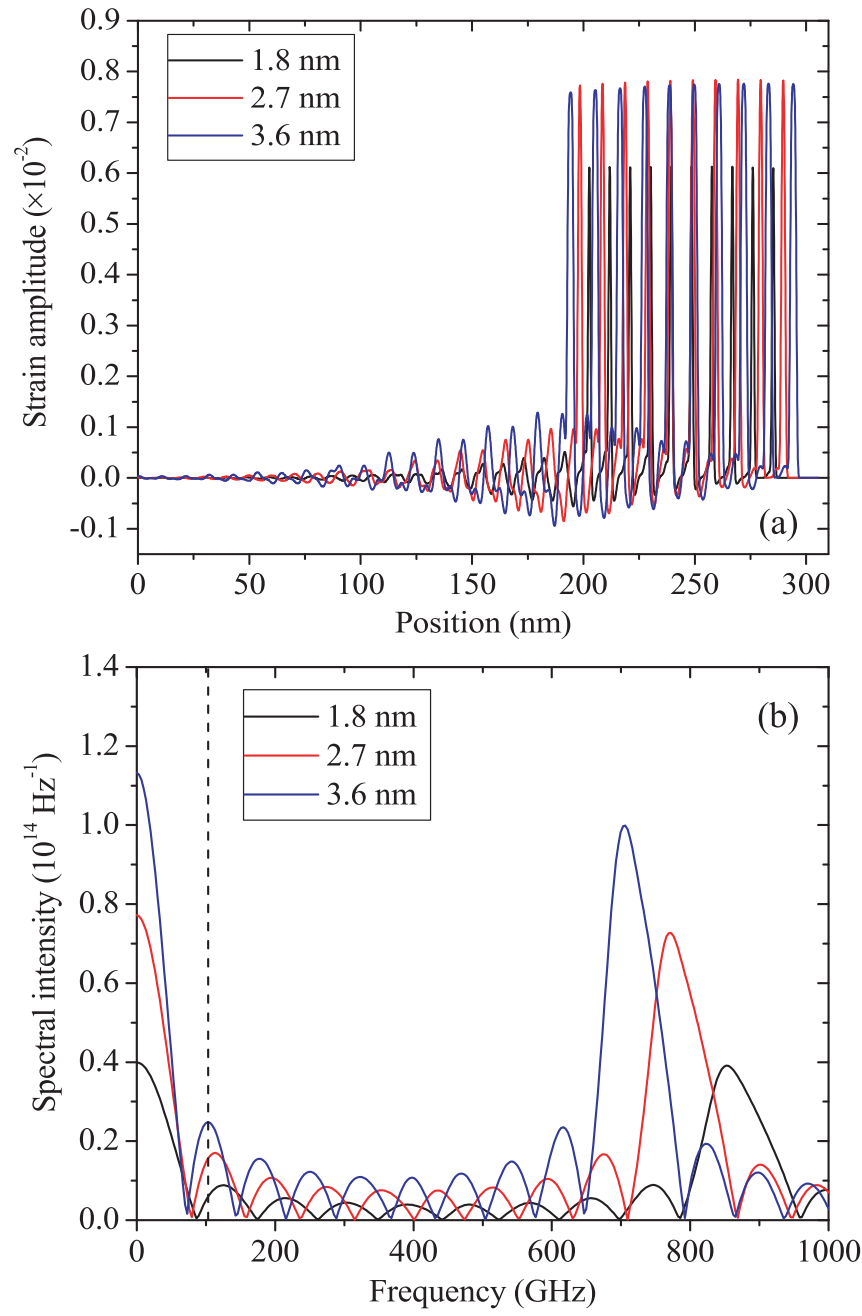

FIG. 7. (Color online) (a) Strain time traces emitted into the buffer layer at $30 \mathrm{ps}$ after propagation, for the three MQW samples. The center of the MQW structure is located at $0 \mathrm{~nm}$. (b) Spectral content of the propagating acoustic wave at saturation of piezoelectric screening, for the three MQW samples. The spectral amplitudes at the Brillouin frequency $f_{0}=103.3 \mathrm{GHz}$ (vertical dashed line) correspond to those given in Fig. 5(d). These results were calculated using maximum coherent strain pulse amplitudes $s_{0}$ of $2.0 \%, 1.8 \%$, and $1.7 \%$ for the respective MQW samples.

\section{CONCLUSIONS}

In conclusion, we have demonstrated that the optical generation of coherent electromagnetic and acoustic $\mathrm{THz}$ emission in $\mathrm{In}_{0.2} \mathrm{Ga}_{0.8} \mathrm{~N} / \mathrm{GaN}$ MQWs has a common origin in the ultrafast screening of the built-in piezoelectric field by polarized photoexcited carriers. The built-in electric field is typically of $3.1 \mathrm{MV} / \mathrm{cm}$, caused by the $2 \%$ lattice mismatch between the QW and barrier material in the $\mathrm{InGaN} / \mathrm{GaN}$, and leads to a spatial separation between electron and hole states as a result of quantum-confined Stark effect. The population of these polarized states by a femtosecond pulse results in ultrafast modification of electric polarization within the QWs and produces a THz electromagnetic pulse which is detected using free-space electro-optic sampling. The $\mathrm{THz}$ electromagnetic emission starts to saturate at fluences around $0.5 \mathrm{~mJ} / \mathrm{cm}^{2}$, long before the optical absorption saturates. Simultaneously, the 
built-in electric field is being screened, removing the balance between mechanical strain in the QW and electrostatic force. As a result, such an "inverse" piezoelectric effect in the QW releases an acoustic strain pulse which is measured by pump-probe spectroscopy. This acoustic pulse has a frequency spectrum peaking in the $0.6-1.0 \mathrm{THz}$ range, consistent with the calculated MQW zone-folded acoustic phonon modes.

We have quantitatively measured the amplitude of the $100 \mathrm{GHz}$ Brillouin oscillations of the strain pulse and demonstrated saturation setting in at fluences around $0.5 \mathrm{~mJ} / \mathrm{cm}^{2}$, at exactly the same pump fluence as the $\mathrm{THz}$ electromagnetic pulse. The spectral intensities of the $100-\mathrm{GHz}$ Brillouin component allow us to derive a value for the generated strain amplitude of around $2 \%$, equal to the lattice mismatch between the QW and barrier material. This demonstrates the complete release of the built-in mechanical strain in the QW as a result of optically-induced dynamical screening of the built-in strain-induced piezoelectric field in the QW.
The THz strain amplitudes measured by us are among the largest observed so far in an ultrafast photoacoustic generation experiments. For comparison, the typical strain amplitudes achieved in thin metal films (the archetypal structures used for picosecond ultrasonics) reach $0.4 \%$, at pump fluences an order of magnitude higher than those used in this work. ${ }^{27} \mathrm{~A}$ prime advantage of InGaN/GaN structures is that it is possible to shape the emitted ultrafast acoustic pulse. ${ }^{24}$ This holds promise for the use of InGaN/GaN MQW systems as high-power photoacoustic sources in the $\mathrm{THz}$ range for ultrafast acoustooptical modulators or the study of ultrafast nonlinear acoustic phenomena and acoustically induced phase transitions.

\section{ACKNOWLEDGMENTS}

C. R. de Kok and P. Jurrius are acknowledged for their technical assistance. This work is a part of the research programme of the "Stichting voor Fundamenteel Onderzoek der Materie (FOM)," which is financially supported by the "Nederlandse Organisatie voor Wetenschappelijk Onderzoek (NWO)." *p.j.s.vancapel@uu.nl

${ }^{1}$ F. Chen, M. C. Cheung, P. M. Sweeney, W. D. Kirkey, M. Furis, and A. N. Cartwright, J. Appl. Phys. 93, 4933 (2003).

${ }^{2}$ D. Turchinovich, P. U. Jepsen, B. S. Monozon, M. Koch, S. Lahmann, U. Rossow, and A. Hangleiter, Phys. Rev. B 68, 241307 (2003).

${ }^{3}$ D. Turchinovich, B. S. Monozon, and P. Uhd Jepsen, J. Appl. Phys. 99, 013510 (2006).

${ }^{4}$ C.-K. Sun, J.-C. Liang, and X.-Y. Yu, Phys. Rev. Lett. 84, 179 (2000).

${ }^{5}$ E. Makarona, B. Daly, J. S. Im, H. J. Maris, and A. Nurmikko, Appl. Phys. Lett. 81, 2791 (2002).

${ }^{6}$ C. Y. Chen, Y. C. Wen, H. P. Chen, T. M. Liu, C. C. Pan, J. I. Chyi, and C. K. Sun, Appl. Phys. Lett. 91, 133101 (2007).

${ }^{7}$ M. R. Armstrong, E. J. Reed, K.-Y. Kim, J. H. Glownia, W. M. Howard, E. L. Pines, and J. C. Roberts, Nat. Phys. 5, 289 (2009).

${ }^{8}$ V. Darakchieva, B. Monemar, and A. Usui, Appl. Phys. Lett. 91, 031911 (2007).

${ }^{9}$ W. Paszkowicz, Powder Diffr. 14, 258 (1999).

${ }^{10}$ J. S. Im, H. Kollmer, J. Off, F. Scholz, and A. Hangleiter, Mater. Sci. Eng. B 59, 315 (1999).

${ }^{11}$ A. Hangleiter, F. Hitzel, S. Lahmann, and U. Rossow, Appl. Phys. Lett. 83, 1169 (2003).

${ }^{12}$ D. A. B. Miller, D. S. Chemla, T. C. Damen, A. C. Gossard, W. Wiegmann, T. H. Wood, and C. A. Burrus, Phys. Rev. Lett. 53, 2173 (1984).

${ }^{13}$ P. C. M. Planken, M. C. Nuss, W. H. Knox, D. A. B. Miller, and K. W. Goossen, Appl. Phys. Lett. 61, 2009 (1992).
${ }^{14}$ D. Turchinovich, Ph.D. thesis, Albert Ludwigs University, Freiburg, 2004.

${ }^{15}$ M. Wraback, H. Shen, A. V. Sampath, C. J. Collins, G. A. Garrett, and W. L. Sarney, Phys. Status Solidi A 202, 790 (2005).

${ }^{16}$ A. Zubilov, Properties of Advanced Semiconductor Materials (Wiley, New York, 2001).

${ }^{17}$ A. Bartels, T. Dekorsy, H. Kurz, and K. Köhler, Phys. Rev. Lett. 82, 1044 (1999).

${ }^{18}$ P. Tyagi, R. R. Cooney, S. L. Sewall, D. M. Sagar, J. I. Saari, and P. Kambhampati, Nano Lett. 10, 3062 (2010).

${ }^{19}$ G. D. Sanders and C. J. Stanton, Phys. Rev. B 74, 205303 (2006).

${ }^{20}$ H. P. Porte, D. Turchinovich, D. G. Cooke, and P. Uhd Jepsen, J. Phys.: Conf. Ser. 193, 012084 (2009).

${ }^{21}$ C. Colvard, T. A. Gant, M. V. Klein, R. Merlin, R. Fischer, H. Morkoc, and A. C. Gossard, Phys. Lett. B 31, 2080 (1985).

${ }^{22}$ S. Tamura, D. C. Hurley, and J. P. Wolfe, Phys. Rev. B 38, 1427 (1988).

${ }^{23}$ F. Hudert, A. Bartels, C. Janke, T. Dekorsy, and K. Köhler, J. Phys. Conf. Ser. 92, 012012 (2007).

${ }^{24}$ C.-T. Yu, K.-H. Lin, C.-L. Hsieh, C.-C. Pan, J. I. Chyi, and C.-K. Sun, Appl. Phys. Lett. 87, 093114 (2005).

${ }^{25}$ H.-N. Lin, R. J. Stoner, H. J. Maris, and J. Tauc, J. Appl. Phys. 69, 3816 (1991).

${ }^{26}$ P. Babilotte, P. Ruello, D. Mounier, T. Pezeril, G. Vaudel, M. Edely, J.-M. Breteau, V. Gusev, and K. Blary, Phys. Rev. B 81, 245207 (2010).

${ }^{27}$ P. J. S. van Capel and J. I. Dijkhuis, Appl. Phys. Lett. 88, 151910 (2006).

${ }^{28}$ P. J. S. van Capel and J. I. Dijkhuis, Phys. Rev. B 81, 144106 (2010). 